\title{
Experimental analysis of the vibration dissipation induced by granular materials included into a 1 degree of freedom oscillator
}

\author{
Antoine Sternberger ${ }^{1,2}$. Jean-Michel Génevaux ${ }^{2}$ • Adrien Pelat $^{2}$
}

Received: date / Accepted: date

\begin{abstract}
The use of granular material is particularly attractive to damp vibrations in hollow structures, due to grains relative motion that induce shocks and friction, interaction grains/structure and possible deformation of the grains. Therefore, granular dampers are of great interest for many application types.However, predictive modelling of the damping performance of a granular material remains particularly difficult. The objective of this experimental work is to evaluate the vibration damping performance of various granular material samples, when inserted in a vertically shaken rigid cavity. This is done by Lissajous' representation of displacement and force time signals, which, applied to this type of system, is a methodological originality allowing a detailed analysis of the overall motion of the grain cluster. The main insights are the identification of links between certain control parameters of the granular material and the vibratory energy dissipated. From Lissajous' representation post-processing, it is shown that dissipation increases linearly with grain mass; at given mass the dissipation increases with grain size; the use of a viscoelastic material notably increases the performance, particularly at low acceleration levels; that the roughness of surfaces in contact with the grains plays a secondary role. Finally, the set of parametric variations give a better physical understanding of the phenomena involved, which gives orientations for future modelling works.
\end{abstract}

\footnotetext{
A. Sternberger

E-mail: antoine.sternberger@irt-jules-verne.fr

${ }^{1}$ IRT Jules Verne, Chemin du Chaffault, 44340 Bouguenais, France

${ }^{2}$ LAUM UMR CNRS 6613, Avenue Olivier Messiaen, 72085 Le Mans, France
}

\section{Introduction}

The vibration control of structures subject to lightening constraints is a major industrial challenge, particularly in the transport industry. Indeed, mass reduction makes it possible to reduce energy consumption but can lead to a significant increase in vibration levels, which leads to premature wear and comfort problems. Classical methods for reducing vibration levels consist of applying to the structure of interest a viscoelastic coating that can eventually be constrained. This technique is very efficient and low-cost but is particularly unsuited to the context of weight reduction because of the very significant mass increase it induces.

In the case of thin structures such as beams, plates or shells, extensive research aims to develop alternative strategies that consist in cleverly fix the mass and stiffness properties in order to control their vibration propagation features. For example, it is possible to use the acoustic black hole effect to trap vibration energy in a small extension area so that it can be dissipated very effectively with a very small amount of viscoelastic material $[28,29]$.Vibration filtering by inserting local resonances [30] or periodic structuring of the mechanical properties of the structures concerned is also very attractive.

The case of hollow structures, referred to in this article, can be addressed by other strategies that take advantage of their natural characteristic of being a container. Typically, the insert of granular material into this type of vibrated structure leads to attractive vibration damping performance. The internal dynamics of the granular material induces in particular many collisions and frictions that dissipate energy. This gives rise to various types of granular dampers whose applications may be considered in aerospace, machinery and 
more recently civil engineering. See the wide literature review work in [2] for an up to date overview of particle dampers technology.

Most studies using granular material to induce vibration attenuation focus on the free vibration of an oscillator on which an initial displacement is imposed. A description of the grain scale contacts is possible for the study of a single grain interacting with the structure where the oscillation is horizontal [7] or vertical [8]. In contrast, Gharib [9] uses a linear grain chain in a resonant system and shows that the number of grains, as well as the mass dispersion of the grain, leads to a change in the vibration attenuation of the system. Mao [10] further increases the complexity of the system by taking a cluster of grains which are either ordered or not.

The vibration of a granular material makes it possible to characterize the dynamic motion of the media. Its motion is non-linear as shown by increasing the acceleration amplitude excitation [11], [12], [13]. These effects involve qualitative observations that can be linked to thermodynamic phases [14]. Applied to resonant structure, Liu [15] has revealed that the non-linear effects induce a non-constant frequency response function (acceleration/force), and a shift in the resonance frequency of the system.

From the numerical point of view, the simulation technique using discrete elements (DEM) allows the position of each grain to be known in a deterministic manner over time. The energy distribution inside the granular material can then be calculated [16]. This simulation technique can also be applied to a forced vibrated granular material whose macroscopic motion is captured to observe variations in excitation conditions on the dissipation in the frequency domain [17]. An experimental and numerical study of a cluster of vibrated grains makes it possible to associate the macroscopic movement of the grains with an apparent mass associated with an amount of energy dissipated by the cluster of grains [18].

Complete analysis of the state of the art, as done in [2], shows that the non-linearities and randomness of the granular material dynamics make it very difficult to establish consistent descriptive theories. To date, the development of a simple analytical model predictive of vibration damping from the parameters of the granular material remains an open question. Such a model would be particularly useful for engineers wishing to design passive damping solutions based on granular material.

The purpose of the work reported in this article is to provide a set of experimental data and related analyses to guide future analytical modelling of the vibration damping produced by a granular material. More precisely, the intention is to establish links between the vibratory energy dissipated and the control parameters of a granular material when it is inserted into a mechanical oscillator at 1 degree of freedom.

The study is built as follows. The experimental set up is described in section 2. As a first step, the measurement of the modal damping coefficient by postprocessing the frequency responses is carried out in section 2.1. Then, a more general technique for measuring the total dissipated energy by means of a Lissajous' representation of time signals is presented in section 2.2 From this second technique, various experimental variations are presented : grains' mass in section 3, grains' diameter in section 4 , grain material in section 5 , grainscontainer contact conditions in section 6 .

\section{Experimental set-up}

The experimental set-up consists of a cavity mounted on two plates in bending acting as a sliding connection and stiffeners, allowing the system to resonate at low frequency (Fig. 1a). A shaker (LDS V406) is used to impose a controlled maximum value of acceleration at the bottom of the cavity. At the same point of the base of the cavity, the acceleration $\gamma_{\text {mes }}$ and the force $F_{\text {mes }}$ are measured using an impedance head (B\&K 8001). The SigLab control box supplies the shaker via an amplifier (PA-100). The granular material studied is placed inside the hollow cavity whose wall has been deliberately made of plexiglass in order to observe the movement of the grains under vibration (Fig. 1b). A circular rigid plate can be moved and fixed inside the cavity to select the effective volume of the cavity.

A control loop keeps the maximum acceleration level constant over a given frequency range (Fig. 1c). To do it, the frequency and a maximum target acceleration $\gamma_{\mathrm{s}}$ are chosen, then a supply voltage is applied to the shaker causing the cavity to vibrate, the acceleration $\gamma_{\text {mes }}$ is measured and compared to the target value, the voltage is adjusted if the measured maximal acceleration is too high or too low and after convergence the acquisition is performed. The last step consists in increasing the frequency and performing this control loop again to have a constant acceleration value over the entire frequency range.

Time measurements can also be performed in order to quantify instantaneous acceleration $\gamma_{\text {mes }}(t)$ and force $F_{\text {mes }}(t)$ over a time of $t=5 \mathrm{~s}$, for a given maximal acceleration. This is done for the resonant frequency which corresponds to phase quadrature between acceleration and force signals. This value depends on the chosen maximal acceleration. 
(a)

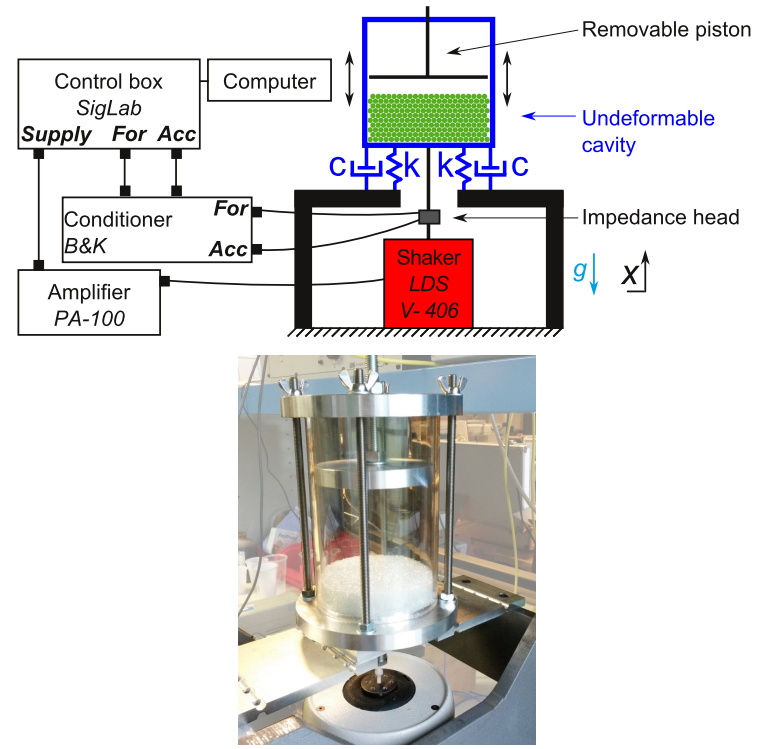

(c)

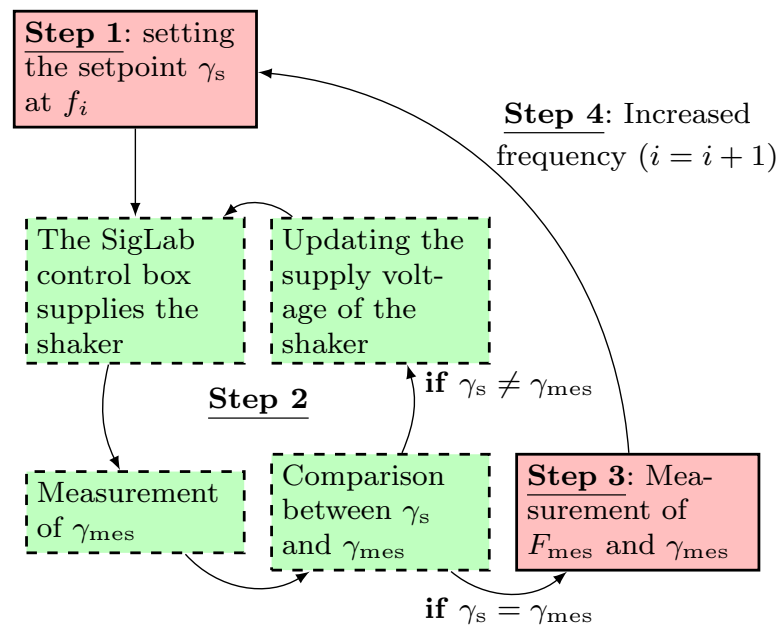

Fig. 1 Experimental setup: (a) sketch of the setup ; (b) picture of the cavity mounted on stiffeners and excited by a shaker ; (c) acceleration control loop as a function of the frequency.

\subsection{Analysis based on Frequency Response Function (FRF)}

The empty cavity mounted on the two plates behaves as a linear oscillator with 1 degree of freedom (1 DoF) in the tested frequency range. The connecting plates are analytically represented by a stiffness and a viscous coefficient. Its parameters are experimentally determined $\left(m_{1}=3.774 \mathrm{~kg}, c_{1}=7 \mathrm{~N} \cdot \mathrm{s} \cdot \mathrm{m}^{-1}\right.$ and $k_{1}=$ $\left.4.85 \cdot 10^{5} \mathrm{~N} \cdot \mathrm{m}^{-1}\right)$ using the kinetic energy of the system in translation for $m_{1}$ and the frequency response for $k_{1}$ and $c_{1}$. Finally, the FRF is defined as the inertance given by the ratio between acceleration and measured force. The vibration measurement contains all the energy loss contributions, notably via the addition of grains. A systematic comparison to the empty reference case is therefore necessary. The granular material used in this first part of the study is glass beads with a diameter of $\varnothing=2 \mathrm{~mm}$ and an added mass of $m_{2}=0.476 \mathrm{~kg}$ (Fig. 2).

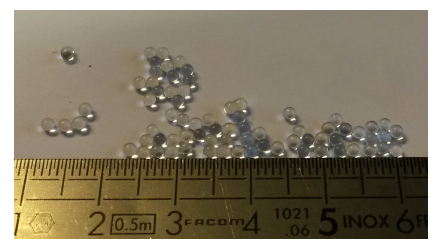

Fig. 2 Glass grains with a diameter of $\varnothing=2 \mathrm{~mm}$.

The added mass effect is highlight by a preliminary experiment comparing the FRF for a clamped added mass and for a granular material of the same mass inserted in the hosted rigid cavity (Fig. 3).

For the clamped added mass configuration, a rather small decrease in the resonance frequency of around $0.4 \%$ (Fig. 3b) and a small increase in the FRF amplitude is observed with the increase of the acceleration level (Fig. 3a): the system can be considered as linear. Conversely, the granular material configuration causes a strong dependence of the FRF to the acceleration level. Note that this non-linearity is not present for $\gamma<$ g: the motion is similar to that of the clamped added mass. For higher acceleration $\gamma>\mathrm{g}$, the amplitude of the FRF significantly decreases (Fig. 3c), the curve widens and the resonance frequency is increased of around $3.8 \%$ (Fig. 3d).

The enlargement of the FRF resonance peak, and therefore the dissipation, can be quantified by a first indicator called equivalent modal damping $(\zeta)$. This indicator requires the complex representation of the module and phase of the frequency response function. To calculate this indicator, a circle must be adjusted at all points considered in order to pass as close as possible to them. This method called "circle-fit" makes it possible to obtain the coordinates of the center of the circle in the complex representation (Fig. 4).

This value approximates a purely viscous energy dissipation [19] p.313 and is calculated according to the formula:

$\zeta=\frac{\omega_{a}^{2}-\omega_{b}^{2}}{2 \omega_{r}\left[\omega_{a} \tan \left(\theta_{a} / 2\right)+\omega_{b} \tan \left(\theta_{b} / 2\right)\right]}$,

with $\omega_{a}$ and $\omega_{b}$ the circular frequencies at $-3 \mathrm{~dB}, \omega_{r}$ the circular frequency resonance, $\theta_{a}$ and $\theta_{b}$, angles in the Nyquist diagram of the FRF.

In the case of glass grains, this coefficient is used as a first approximation of the energy loss. In our case, four experiments are set up to compare different configurations according to the acceleration amplitude (Fig. 5). 
(a)

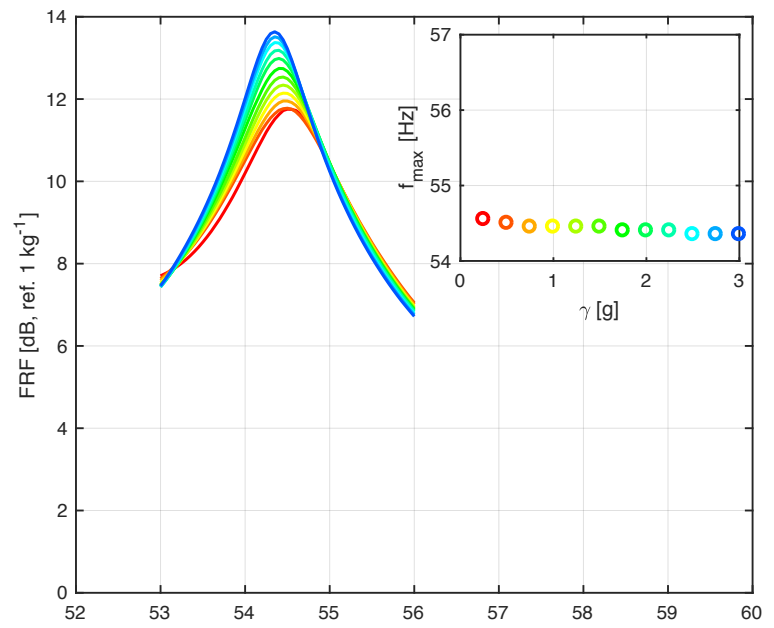

(b)

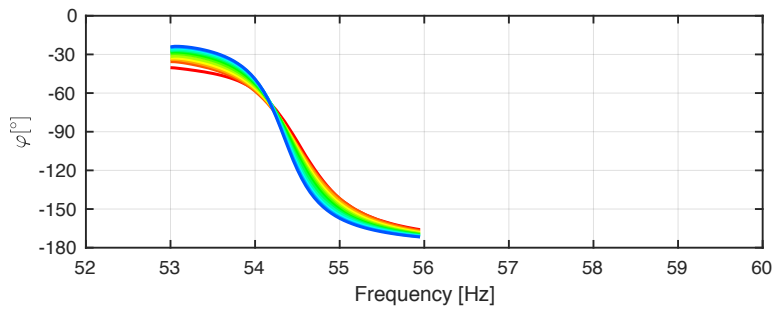

(c)

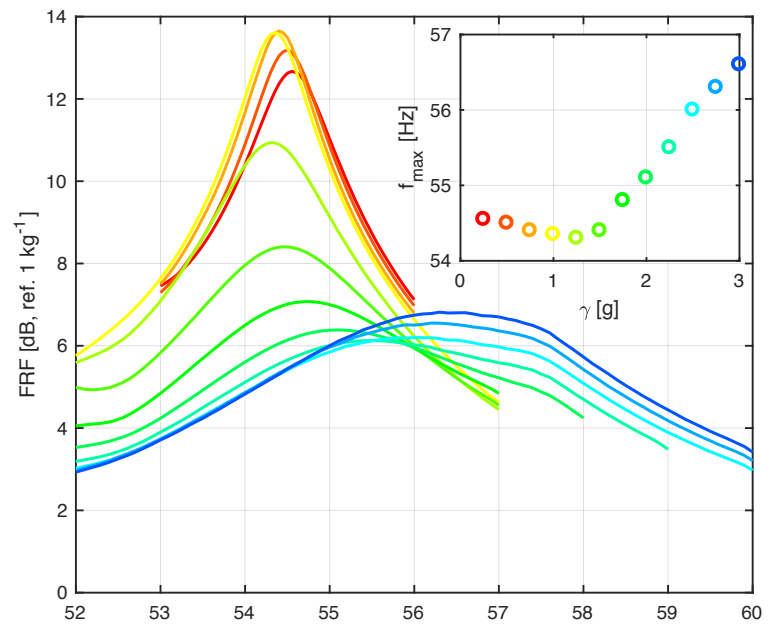

(d)

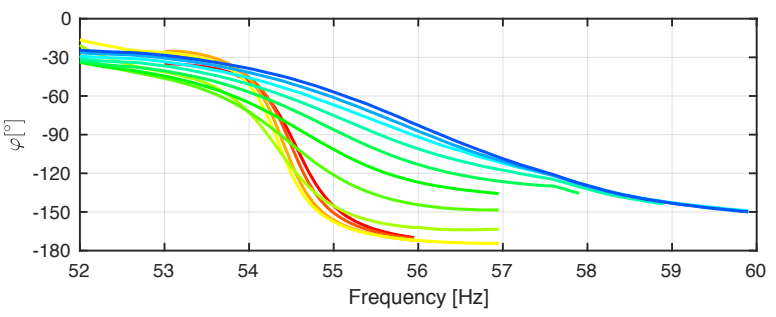

Fig. 3 Experimental Frequency Response Function (FRF) of the host system with an added clamped rigid mass : (a) modulus and (b) phase ; and with an embedded granular material : (c) modulus and (d) phase. The colors of the curvesfrom blue to red denotes the imposed maximal acceleration. Insets in (a) and (c) show for each acceleration's case the corresponding value of the resonant frequency.
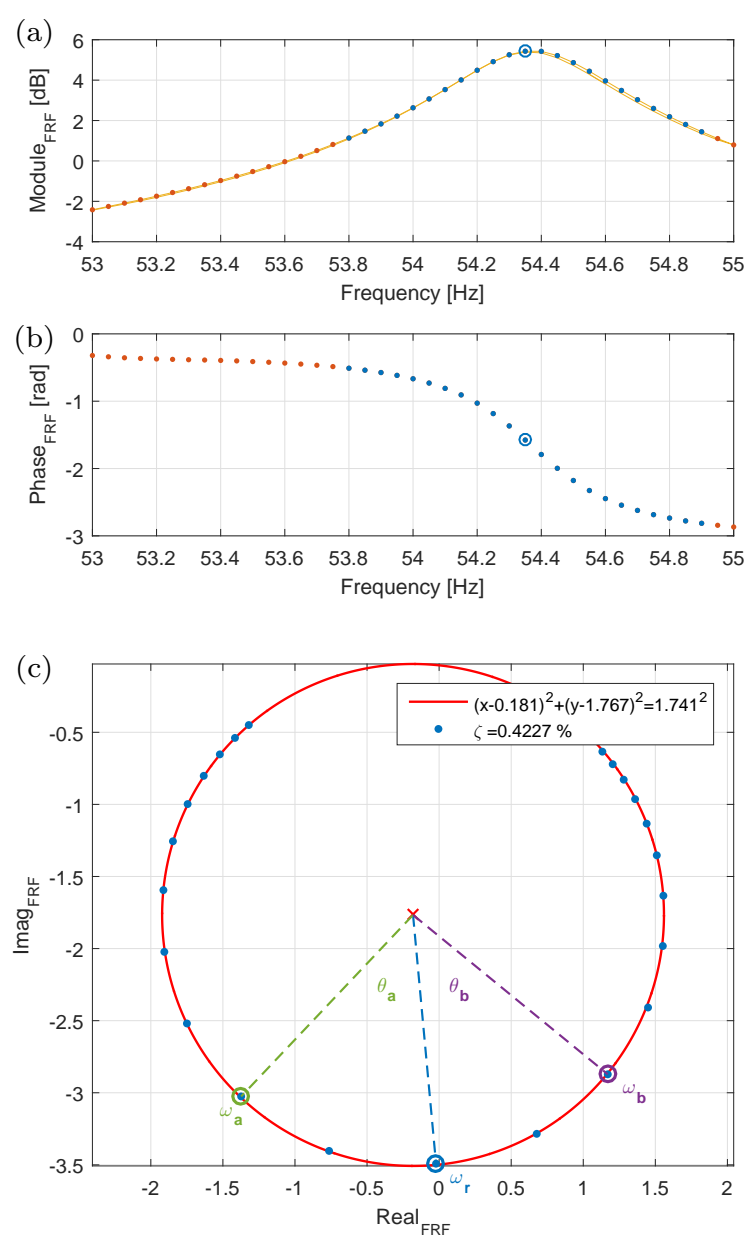

Fig. 4 Representation of the Frequency Response Function (FRF): (a) modulus ; (b) phase ; (c) complex representation.

First of all, the cavity is studied without treatment and with the addition of a fixed non-deformable mass in order to have two reference cases. Then, the addition of a granular material consisting of glass grains in a compact and free configuration is applied. The uncertainty on $E_{L}$ is calculated from the deviation from the average and is given for a 95\% confidence level [31] (see the appendix for the main calculation steps).

For the two reference cases, the coefficients $\zeta$ remain independent of the acceleration amplitude. The increase in vibration energy therefore has no effect on the property of these configurations to attenuate vibrations. For grains compacted by moving the removable piston in order to press the grains an let only a cavity volume that corresponds to the volume of grains, the dissipative behaviour is identical to that of the fixed non-deformable mass. The grains are not free to move and no added dissipation appears. If the piston is removed, the volume of granular material presents a free surface, and the higher the acceleration, the greater the 


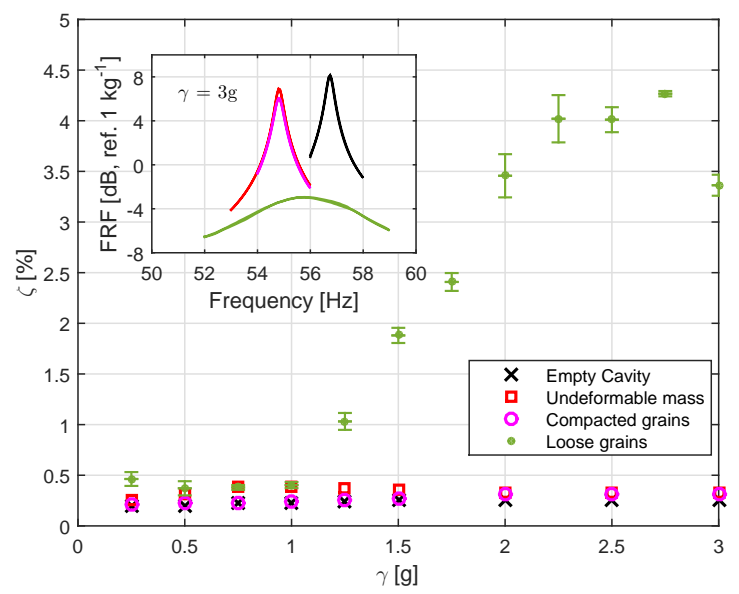

Fig. 5 Modal damping coefficient $(\zeta)$ as a function of the acceleration $(\gamma)$ for several vibration treatments.

dissipation. This difference can be seen in the zoomed figure on Fig. 5 representing the FRF for the four configurations at an acceleration of $\gamma=3 \mathrm{~g}$, the curve with the lowest amplitude is that corresponding to the granular material with a free surface above. A more precise observation of the evolution of the modal damping coefficient $(\zeta)$ shows the presence of a threshold at $\gamma=\mathrm{g}$, value from which the dissipation increases strongly until a factor of 10 compared to the fixed non-deformable mass. The increase in interactions between grains may be a consistent explanation but cannot be observed by means of this frequency representation. A study based on a temporal representation of the acquired signals is therefore implemented.

\subsection{Analysis based on time signals}

To characterize in more details the motion and related dissipation of the cluster of grains, the acceleration $\gamma_{\text {mes }}$, displacement $x=-\omega^{2} \gamma_{\text {mes }}$ and force $F_{\text {mes }}$ time signals are plotted in Fig. 6a and Fig. 6b. The system is excited at the resonance frequency so that displacement and force are in phase quadrature. A phase difference The second representation of time signals in Fig. 6c, called Lissajous' representation, plots the signals in the force-displacement plane. Each complete lap of this diagram corresponds to a single period. In the displayed example, 259 period are involved so that an overlap can be seen.

These representations show that the experimental signals are not perfectly sinusoidal, so dissipation cannot be due exclusively to viscous damping, but to other mechanisms too. Remarkable points can be observed on the different graphs in Fig. 6. When the instantaneous acceleration reaches the value $\gamma=-\mathrm{g}$ (point TK), the (a)

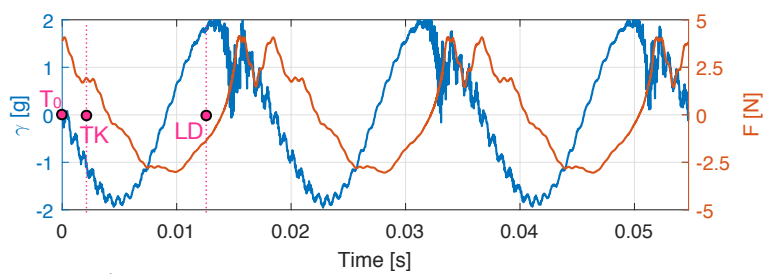

(b)

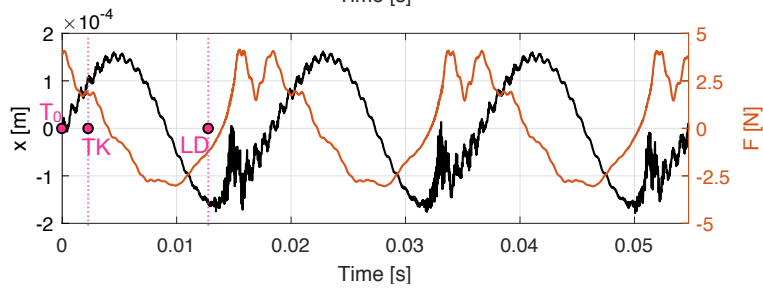

(C)

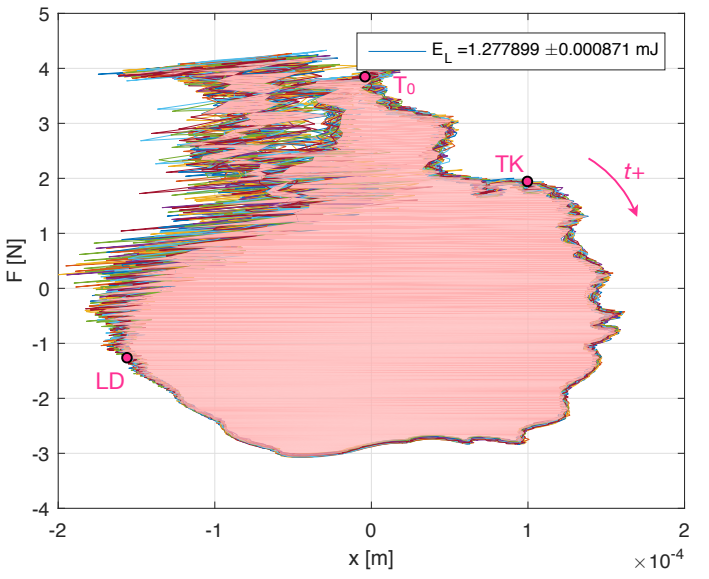

Fig. 6 Experimental measurement signals of the cavity filled with glass grains $(\varnothing=2 \mathrm{~mm}-\gamma=2 \mathrm{~g}$ ): (a) time signals of acceleration (blue) and force (red) ; (b) time signals of displacement (blue) and force (red) ; (c) superposition of Lissajous' representation representing the force as a function of the displacement.

cluster of grains takes off from the base of the cavity. A high speed video shows this motion (video of the experiment carried out: [20]). This leads to a decrease in the value of the force, the acceleration being controlled. There is a substantial phase shift between the two signals even after take-off. This variation reflects part of the internal dissipation due to an equivalent viscous damping corresponding to the large amount of contacts inside the granular material. It can be explained by the fact that, during the flight, the grains on the side of the cluster remain in contact with the side wall of the cavity. A periodic singularity on the displacement signal (point LD) is due to the grain landing mechanism at the base of the cavity. This particular point is correlated with a sudden increase in the force signal. The following fluctuations in the force signal corresponds to a mode of the rod connecting the shaker to the base of the cavity. Indeed, to avoid having a hyperstatic system, this rod made of soft material keeps the guidance 
of the cavity ensured by the two plates and not by the shaker.

The Lissajous' representation allows to calculate the associated energy loss $E_{L}$ :

$E_{L}=\frac{1}{N_{\text {cycles }}} \oint_{\text {cycles }} F \mathrm{~d} x$

where $N_{\text {cycles }}$ is the number of measured cycle/period, $F$ the measured force and $x$ the displacement deducted from the measured acceleration. The energy loss per cycle $E_{L}$ is the calculated internal area of the curve (in Joules). The uncertainty on $E_{L}$ is estimated from the deviation from the average and is given for $95 \%$ confidence level. The high precision of the measure of $E_{L}$ is due to the high number (259) of cycles considered to calculate the average value.

For the empty cavity and the clamped rigid mass cases, the Lissajous' representation is an ellipse (not shown here for conciseness) which corresponds to a purely viscous damping. For granular material with a free surface, the shape of the cycle is more complex (Fig. 6c) and the associated energy loss $E_{L}$ non-linearly increases with the maximal acceleration (Fig. 7). Under the $\gamma=$ $|\mathrm{g}|$ threshold, the loose grains configuration does not add a significant dissipation. Note for example that for $\gamma=3 \mathrm{~g}, m_{2}=0.476 \mathrm{~kg}, \varnothing=2 \mathrm{~mm}$, glass grains, the dissipation is 10 times greater than with the clamped added mass.

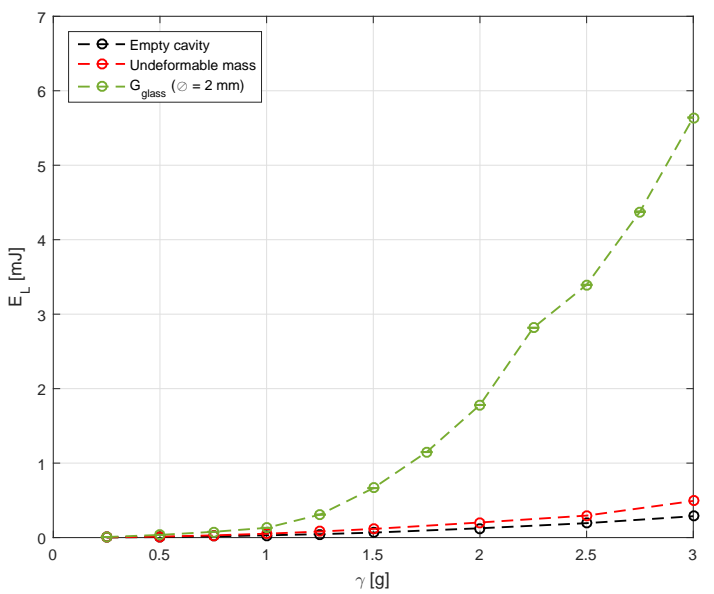

Fig. 7 Energy loss $E_{L}$ measured as a function of the acceleration for several treatments with a given mass $m_{2}=0.476 \mathrm{~kg}$.

The following sections aim to quantify the influence of the different control parameters of the granular material on dissipation.

\section{Variation in the grain mass}

The objective of this parametric variation is to evaluate how the dissipated energy depends on the grain mass $m_{2}$ contained in the cavity. The grains are made of glass so as to neglect viscoelastic losses. All grains have the same $2 \mathrm{~mm}$ diameter for all experiments. The piston is raised so that the upper surface of the grains is free (loose grains configuration). For each tested mass, a reference experiment is done with the same mass of a rigid material (aluminium block) clamped at the bottom of the cavity.

First, in Fig. 8 the energy dissipated for 3 grain masses is measured as a function of the imposed maximum acceleration. The results show that whatever the acceleration, the larger the grain mass, the more energy is dissipated. On the other hand, the energy dissipated does not depend on mass in the clamped mass configurations, as expected.

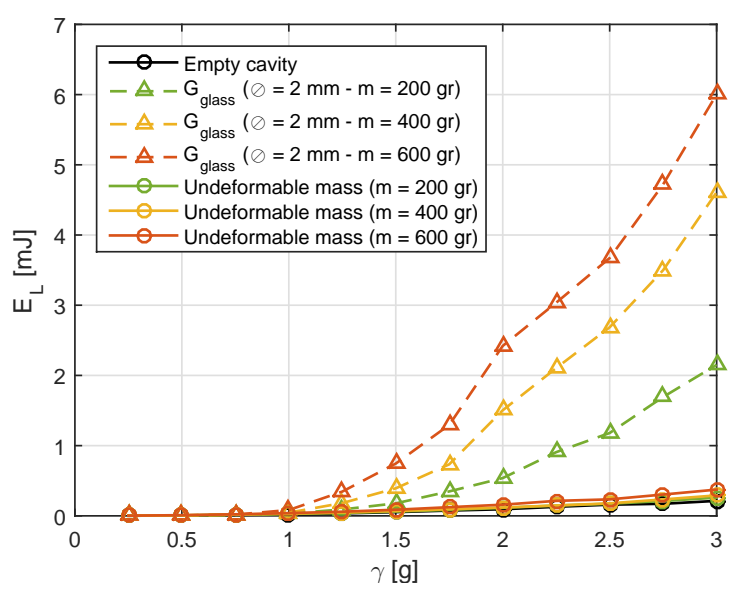

Fig. 8 Energy loss $E_{L}$ measured as a function of the acceleration for a few grain mass inserted into the cavity.

A second study in Fig. 9 is then carried out to identify in more detail the energy loss and resonance frequency as functions of the grain mass. The mass variation is finer, from $25 \mathrm{gr}$ to $600 \mathrm{gr}$ with a $25 \mathrm{gr}$ step, and the maximum acceleration is fixed to $\gamma=2 \mathrm{~g}$. The results show that the decrease in resonance frequency from $f_{0}$ without grains to $f_{m}$ with an added grain mass $m_{2}$, corresponds exactly to the added mass effect $f_{m} / f_{0}=\sqrt{m_{1} /\left(m_{1}+m_{2}\right)}$. Moreover, the energy loss increases linearly with grain mass: $E_{L}=a m_{2}+b$, with $a=3.84 \pm 0.13 \mathrm{~mJ} /($ cycle $\cdot \mathrm{kg})$ and $b=-0.005 \pm$ $0.046 \mathrm{~mJ} /$ cycle (confidence level 95\%). Due to the value of $a$, even for a small mass of grain, the added dissipation is significant compared to the value of $b$ which represents the dissipation of the empty host system. 
Anyway, the linearity between the damping and the added mass permits to explore the other parameters (diameter, viscoelasticity, grains-cavity contact conditions) from a single given mass.

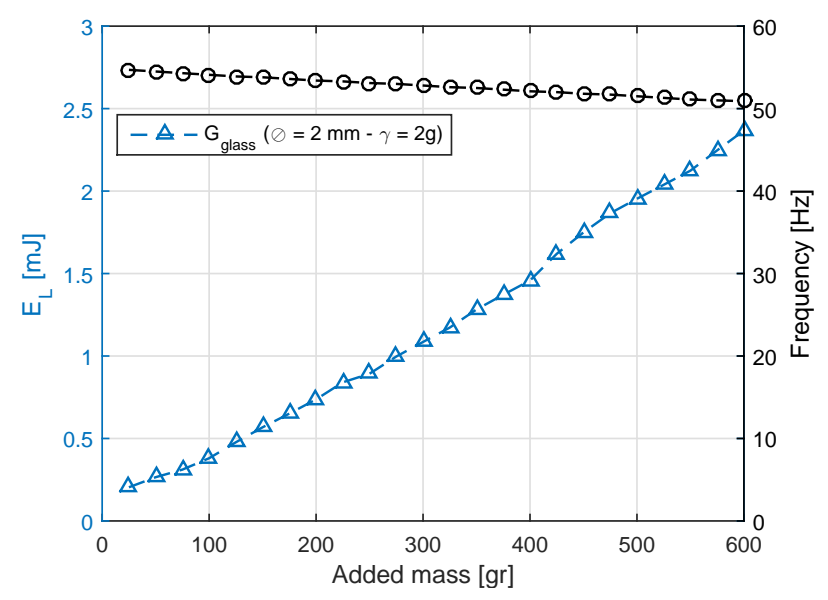

Fig. 9 Energy loss $E_{L}$ (blue triangles) and the associated resonance frequency black circles as functions of the added grain mass for $\gamma=2 \mathrm{~g}$.

\section{Variation in grain diameter}

In the previous experiment, increasing the mass leads to increase simultaneously the number of contacts. To isolate the effect of the number of contacts on damping, this section presents results obtained at constant mass and grain material for different grain diameters.

At given mass $\left(m_{2}=0.476 \mathrm{~kg}\right)$ and material $\left(\rho_{\text {glass }}=\right.$ $2530 \mathrm{~kg} \cdot \mathrm{m}^{-3}$ ), the number of grains is estimated in Tab. 1 from their diameter.

\begin{tabular}{|ll|}
\hline$\varnothing=2 \mathrm{~mm}$ & $N_{\text {grains }} \approx 44900$ \\
\hline$\varnothing=1 \mathrm{~mm}$ & $N_{\text {grains }} \approx 359000$ \\
\hline$\varnothing=0.2 \mathrm{~mm}$ & $N_{\text {grains }} \approx 44900000$ \\
\hline
\end{tabular}

Table 1 Variation in the number of grains inside the cavity according to the diameter with $m_{2}=0.476 \mathrm{~kg}$.

Fig. 10 shows the time signals and Lissajous' representation of the force and displacement in the case $\varnothing$ $=0.2 \mathrm{~mm}$ and $\gamma=2 \mathrm{~g}$.

First, the Lissajous' representation shows a smoother overall behaviour. Take-off (point TK) and landing (point LD) phenomena of the cluster of grains are still observed but without rod oscillations as previously seen in Fig. 6c. Indeed, due to the smaller size of grains, the impact on landing is slower and therefore less intense than with larger grains. As a consequence, the (a)

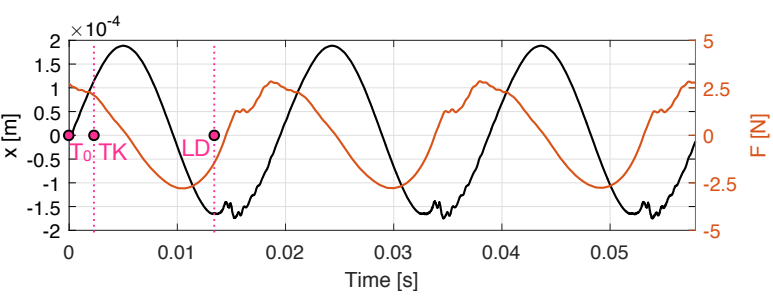

(b)

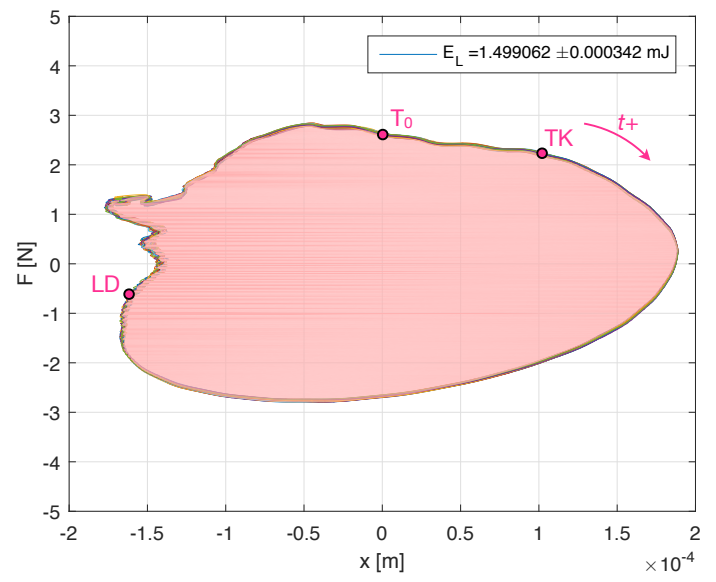

Fig. 10 Experimental measurement signals of the cavity filled with glass grains $(\varnothing=0.2 \mathrm{~mm}-\gamma=2 \mathrm{~g}$ ): (a) time signals of displacement (blue) and force (red) ; (b) superposition of Lissajous' representation representing the force as a function of the displacement.

representative surface mainly shows an ellipse between points TK and LD extended by a landing surface that increases dissipation. In addition, the 259 cycles are almost superimposed, which indicates that the repeatability is very high compared to that obtained for larger diameter grains, even during the landing phase (point 3).

Fig. 11 shows that the larger the grains, the greater the dissipation. An increase of $20 \%$ of dissipation from $\varnothing=0.2 \mathrm{~mm}$ to $\varnothing=2 \mathrm{~mm}$ is observed. At constant macroscopic kinetic energy (constant mass, controlled acceleration), decreasing the number of grains increases the kinetic energy of each grain so that shocks are more energetic. As a result, it is therefore more efficient to maximize the energy dissipated per shock rather than the number of shocks. This is consistent with the nonlinear dependence of dissipation with the kinetic energy of two colliding particles. Moreover, the viscous effects of air become significant for small diameters, transforming the discrete granular material into an equivalent fluid with macroscopic motion.

To complete the analysis, the FRFs plotted in Fig. 12 confirm that at the macroscopic scale, non-linear mechanisms are greatly reduced when the grain diameter is small. Indeed, the increase in resonance frequency becomes negligible with $\varnothing=0.2 \mathrm{~mm}$ while it is $3.57 \%$ with $\varnothing=2 \mathrm{~mm}$. In this case, the granular material is 


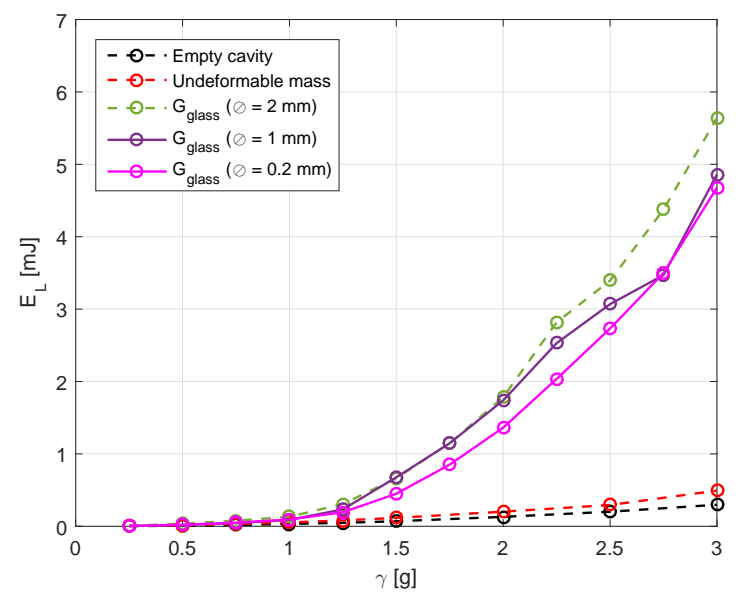

Fig. 11 Energy loss $E_{L}$ measured as a function of the acceleration for several glass grain diameter.

similar to an equivalent viscous fluid (elliptical shape of Lissajous' representation in Fig. 10) that provides less loss.

Note that for technical reasons the stiffeners had to be replaced for these measurements. As a result, the resonance frequency at which measurements are made is very slightly reduced. This does not affect the high quality of the signals and does not disrupt the overall work strategy.

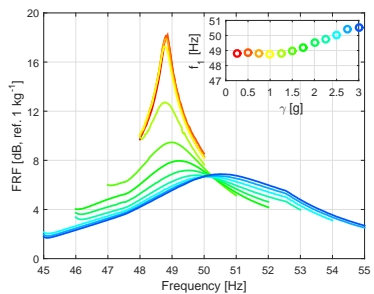

(a)

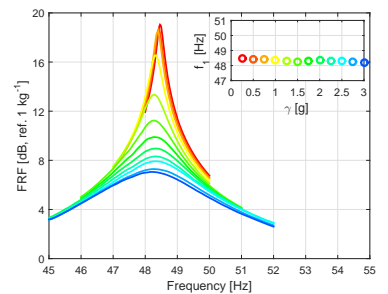

(b)
Fig. 12 FRF for several grains diameter: (a) $\varnothing=2 \mathrm{~mm}$; (b) $\varnothing=0.2 \mathrm{~mm}$.

\section{Additional damping due to inelastic contacts with viscoelastic grains}

In all results presented so far, no dissipation is produced for accelerations $\gamma<1 \mathrm{~g}$ due to no relative motion between glass grains (assumed rigid). However, there are many vibration mitigation applications in this range of acceleration levels. The use of viscoelastic grains that are able to induce dissipation in addition to that induced by collisions is then considered in this section.

The used grains (Fig. 13) are hollow spherical shells made of epoxy with mineral charges, manufactured by
ATECA company. A detailed characterization has been done in $[22,23]$. Only the basic mechanical parameters are recalled here, among the results from numerous tests : Young's modulus $E=600 \mathrm{MPa}$, density $\rho=1985 \mathrm{~kg} \cdot \mathrm{m}^{3}$, Poisson ratio $\nu=0.015$, mean external diameter $\varnothing \approx 2 \mathrm{~mm}$. These grains have been used for damping honeycomb structures [21] or cryogenic tanks [24].

The very small ball that can be observed inside the grain is the residue of an initial polystyrene filling sphere around which epoxy is deposited. When heating, this filling then retracts, leading to the spherical shell. The mass of this residue is negligible so that its contribution to dissipation is ignored compared to the other effects involved.

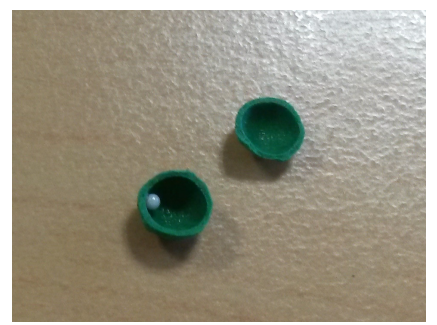

Fig. 13 Viscoelastic hollow grains (ref. E171101, ATECA, France).

A grain mass $m_{2}=0.470 \mathrm{~kg}$ is used according to 3 configurations:

1. "loose grains": the piston is completely raised so that the upper surface of the grains is free

2. "compacted grains": the piston is adjusted to the height of the upper surface of the grains, without applying mechanical stress to them.

3. "loaded grains": the piston is no longer rigidly attached to the cavity. In statics, it stands on the grain upper surface, applying a spatially uniform pressure due to its own weight. In dynamics, the it acts as a mass, the grains as a stiffness, resulting in an secondary 1DOF oscillator coupled to the host system.

Fig. 14 shows typical signals obtained for the configuration "loose grains". As already observed previously, the grains take off (point TK), are in parabolic flight, then land (point LD), which causes a peak of effort followed by rod oscillations as previously. Moreover, when the cavity goes down (approximately $5<t<15 \mathrm{~ms}$ for the first period), a series of noisy patterns appear on the displacement signal. They are attributed to stickslip between the grains and the lateral walls, which can sometimes suddenly reduce the force measured on the cavity. 
(a)

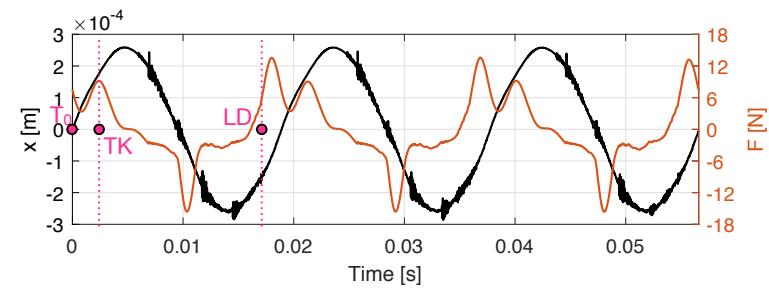

(b)

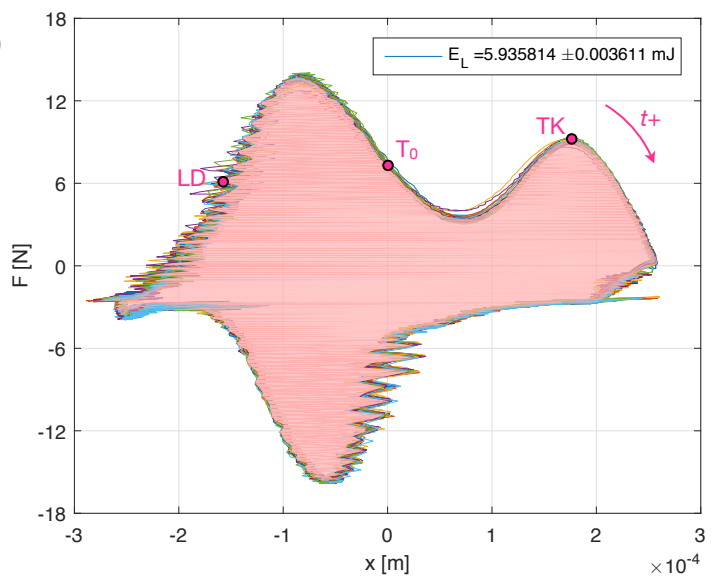

Fig. 14 Experimental measurement signals of the cavity filled with viscoelastic hollow grains $(\varnothing=2 \mathrm{~mm}-\gamma=3 \mathrm{~g})$ : (a) time signals of displacement (blue) and force (red) ; (b) superposition of Lissajous' representation representing the force as a function of the displacement.

Fig. 15 shows that the "loose grains" configuration, which combines collision losses and viscoelastic losses, leads to a significant increase in the energy loss. In particular, the dissipation is multiplied by 4 when $\gamma<1$ g.

On the other hand, the "compacted grains" configuration, which keeps the grains together and thus prevents collisions, induces little dissipation. Since the grain mass is only $12 \%$ of the mass of the cavity, the viscoelastic losses remain low.

More interestingly, the "loaded grains" configuration shows intermediate results. Since the natural frequency of the piston-grain oscillator is very low compared to that of the host system at which the motion is forced, the piston is decoupled and therefore motionless. Consequently, the oscillations of the cavity lead to compression waves in the grain bulk, which then dissipate some energy. However, an acceleration limit value $\gamma>2 \mathrm{~g}$ is present beyond which the mass of the removable piston interacts very strongly with the cavity walls. The induced shocks do not allow the energy dissipated to be measured.

\section{Modification of the surface properties}

Previous measurements have shown that grain-wall contact conditions can have an impact on the behaviour of

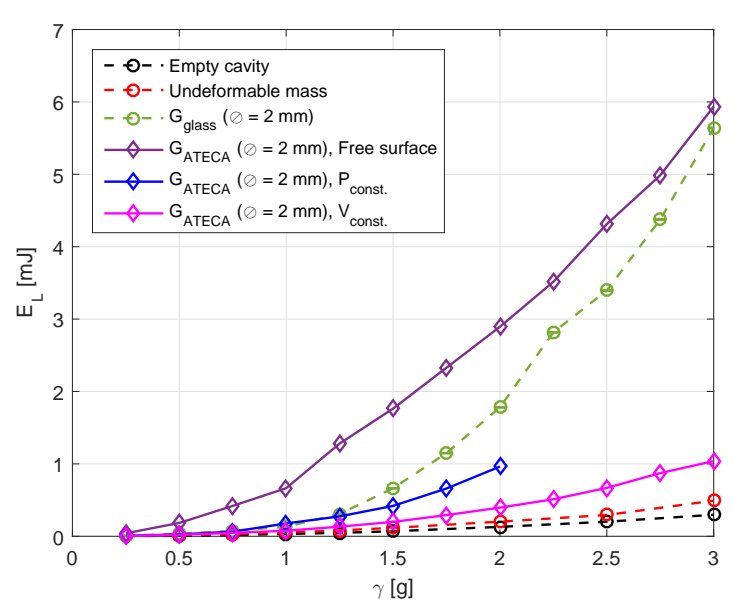

Fig. 15 Energy loss $E_{L}$ measured as a function of the acceleration for the 3 configurations of viscoelastic hollow grains.

the particle damper. The objective of this section is to evaluate the interest of coating the cavity walls on the energy loss.

\subsection{Additional friction on lateral walls}

In this first study, the objective is to increase grain-wall friction during the parabolic flight phase of the grains by sticking abrasive paper to the lateral walls. The 4 used roughnesses $[0.125 ; 0.201 ; 0.201 ; 0.269 ; 0.425] \mathrm{mm}$ correspond to international standards $[P 120 ; P 80 ; P 60 ; P 40]$, respectively. A pcture of the coted cavity is shown in Fig. 16 The grain diameter is intentionally chosen to correspond to the medium paper grain size, i.e. $\varnothing=$ $0.2 \mathrm{~mm}$.

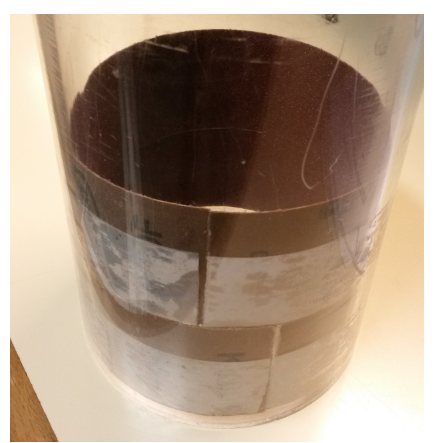

Fig. 16 Picture of the cavity coated by abrasive paper to enhance friction on lateral walls.

Observing the synthesis of lost energy enables to compare the influence of different treatments. Fig. 17 shows that finally the granularity of the lateral wall has no major effect on the energy loss. Thus, the energy loss 
appears to be mainly driven by internal interactions in the bulk of the granular material.

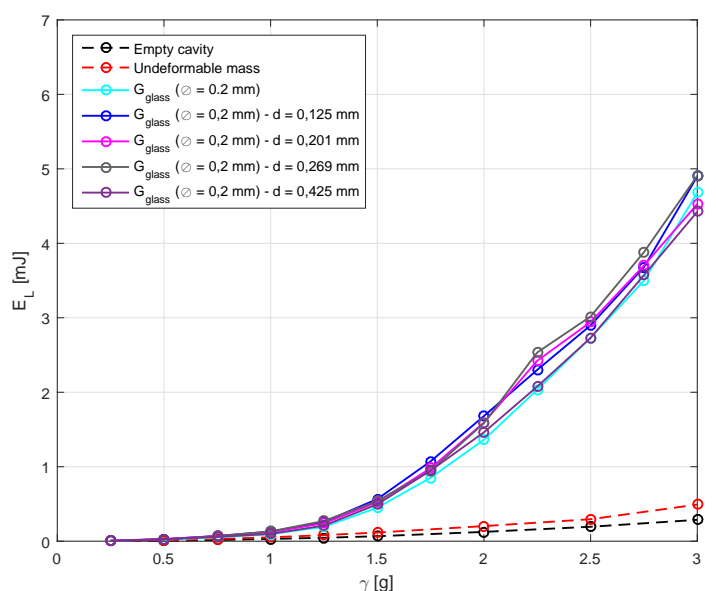

Fig. 17 Energy loss $E_{L}$ measured as a function of the acceleration for several abrasive coating on the lateral walls.

6.2 Addition of a soft surface at the bottom of the cavity

In this second study, the objective is to maximize the energy loss when the grains land. To do this, a felt is clamped around the bottom contour of the cavity in order to help in damping the normal shocks of the grains (Fig. 18). The energy dissipation properties of this type of material have already been notably studied in the case of vibration mitigation of machines [25,26]. The study is carried out with grains $\varnothing=2 \mathrm{~mm}$. The side wall is kept without treatment.

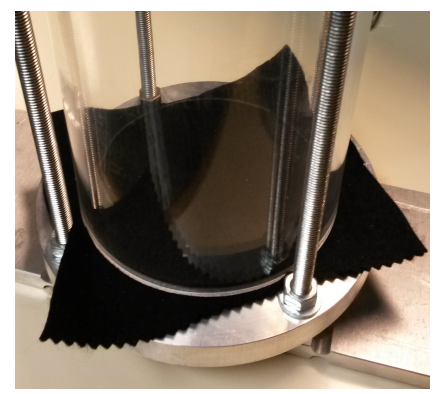

Fig. 18 Picture of the cavity coated at its bottom by a soft surface.

At low accelerations $(\gamma<1.5 \mathrm{~g})$, the felt layer that acts as a viscoelastic layer able to increase the motion of the first layer of grains and so into the bulk. As a result the energy loss can be significantly increased.

For $\gamma \in[1.75,2.25] \mathrm{g}$, the motion of the grains displays bursts (see the video in [27]), which saturates the force sensor and the accelerometer so that the dissipation can not be mesured.

For $\gamma \geq 2.5 \mathrm{~g}$, the dissipation becomes less important in this case. The equivalent viscoelasticity property is no longer validated. For these acceleration values, the grains do not land over the current oscillation period but the next one. Making this landing softer results in a reduction in lost energy.

The difference in dissipative behaviour as a function of acceleration in the case of the coating at the base of the cavity was not further investigated. Nevertheless, the amount of energy lost using glass grains can be maximized by significantly varying the surface condition of the container.

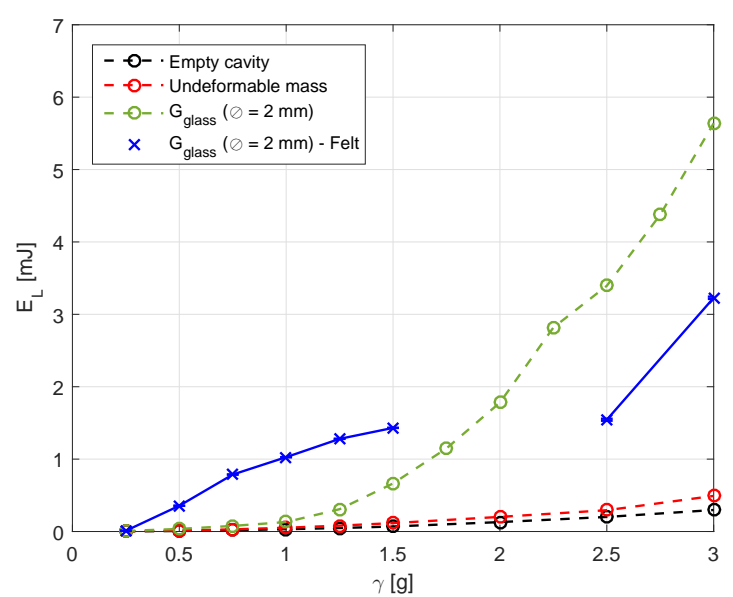

Fig. 19 Energy loss $E_{L}$ measured as a function of the acceleration for a soft base at the bottom of the cavity.

\section{Conclusion}

An experimental setup is designed to evaluate the vibration dissipation perfromances of a granular materials inserted in a rigid cavity, acting as a 1 degree of freedom oscillator. When exciting the resulting system at its resonance frequency, Lissajous' representations of force and displacement time signals are done in order to identify the underlying dissipative mechanisms and quantify the total energy loss per cycle.

From a series of parametric variations, the following conclusions can be figured out :

1. The energy loss is proportional to the mass of embeded grain. The proportionality coefficient is pre- 
cisely estimated in the case of glass grains with a diameter of $2 \mathrm{~mm}$. A more detailed analysis of the evolution of this coefficient as a function of the material parameters is a direct perspective of this result.

2. For a given grain mass, maximizing grain size maximizes the energy loss. When the grains become very small, the contact non-linearities are greatly reduced and the granular material behaves as an equivalent viscous fluid that causes less losses.

3. At a given grain mass, the use of a viscoelastic material significantly increases the energy loss. This increase is particularly interesting at low acceleration levels $(\gamma<1 \mathrm{~g})$ where the internal dynamics of the granular material does not induce measurable dissipative non-linear mechanisms.

4. Introducing friction to the lateral walls does not increase the energy dissipated. On the other hand, introducing viscoelastic effects on the bottom of the cavity can amplify the gain cluster motion and thus increase losses.

This conclusions bring more physical understanding on the links between the control parameters of the grains (at a micro-scale) and the dissipation poperties of a cluster of grains (at macro-scale). Firther works related to modeling could typically be oriented by this conclusions and would aim to obtain engineering tools usefull for the design of granular dampers.

\section{Appendix - uncertainty in measurement}

The measurement uncertainty was performed in relation to the deviation of the dissipated energy per cycle from the mean value [31]. The variance is then calculated to extract the dispersion of the measured values:

$s_{\mathrm{m}}^{2}=\frac{1}{n-1} \sum_{j=1}^{n}\left(q_{j}-\bar{q}\right)^{2}$,

where $n$ is the number of measurements, $\bar{q}$ the mean number of the measurement, $q_{j}$ the value of the considered measure at the place $j$. The followed expression of uncertainty is then found as:

$u_{\mathrm{m}}=\frac{s_{\mathrm{m}}}{\sqrt{n}}$.

An enlargement factor can be found that makes it possible to represent a statistical distribution of the values around the measured average value. The uncertainty $u_{\mathrm{m}}$ is then multiplied by a coefficient $k=2$ allowing the statistical values to have a $95 \%$ chance of being in the interval:

$U_{\mathrm{m}}=u_{\mathrm{m}} \cdot k$,

where $U_{\mathrm{m}}$ is the enlarged uncertainty.

Acknowledgements The authors would like to thank Stphane Job (Supmeca, France) for the fruitful discussions, Herv Mzire for his help in the setup design, Julien Nicolas who manufactured the setup and Mathieu Scail for his fruitfull contribution in the experiments.

This study is part of the VIBROLEG project managed by IRT Jules Verne (French Institute of Research and Technology in Advanced Manufacturing Technologies for Composite, Metallic and Hybrid Structures).

\section{References}

1. M. Ott et al., The effectiveness of particle damping for use on vertical surfaces, 43rd International Congress on Noise Control Engineering, Santa Catarina, BRAZIL (2014)

2. Z. Lu et al., Particle impact dampers: Past, present, and future. Struct Control Health Monit., 25 (2017)

3. Lu et al., An experimental Investigation into the use of buffered particle dampers, World Conference on Earthquake Engineering, Lisboa, PORTUGAL (2012)

4. Duvigneau, F. and Koch, S. and Woschke, E. and Gabbert, U., An effective vibration reduction concept for automotive applications based on granular-filled cavities, Journal of Vibration and Control, 24(1), 73-82 (2018)

5. Heckel et al., Granular dampers for the reduction of vibrations of an oscillatory saw, Physica A: Statistical Mechanics and its Applications, 391, 4442 - 4447 (2012)

6. P. Lieber and D. P. Jensen, An Acceleration Damper: Development, Design and Some Applications, Transactions of the American Society of Mechanical Engineers, 67, 523 530 (1945)

7. C. N. Bapat and S. Sankar, Single unit impact damper in free and forced vibration, Journal of Sound and Vibration, 99, 85 - 94 (1985)

8. M. Trigui et al., An experimental study of a multi-particle impact damper, Journal of Mechanical Engineering Science, 223, 2029-2038 (2009)

9. M. Gharib and S. Ghani, Free vibration analysis of linear particle chain impact damper, Journal of Sound and Vibration, 332, 6254 - 6264 (2013)

10. K. Mao et al., Simulation and Characterization of Particle Damping in Transient Vibrations, Journal of Vibration and Acoustics - Transactions of the ASME, 126, 202 - 211 (2004)

11. A. Sternberger et al., Experimental added modal damping induced by confined granular media on a single degree of freedom system, EPJ Web Conf., 140, 10004 (2017)

12. F. Melo, Hexagons, Kinks, and Disorder in Oscillated Granular Layers, Phys. Rev. Lett., 75, 3838 - 3841 (1995)

13. S. Sen, Thermalizing an impulse, Physica A: Statistical Mechanics and its Applications, 299, 551 - 558 (2001)

14. K. Roeller, Liquid-Gas Phase Separation in Confined Vibrated Dry Granular Matter, Physical Review Letters, 107, 048002 (2011)

15. W. Liu, The dynamic characterisation of disk geometry particle dampers, Journal of Sound and Vibration, 280, 849 - $861(2005)$ 
16. K. Mao, DEM simulation of particle damping, Powder Technology, 142, 154 - 165 (2004)

17. M. Sanchez, Nonlinear dynamic analysis of an optimal particle damper, Journal of Sound and Vibration, 332, 2070 - 2080 (2013)

18. M. Masmoudi, Experimental and numerical investigations of dissipation mechanisms in particle dampers, Granular Matter, 18, 71 (2016)

19. D.J. Ewins, Modal testing: theory, practice and application - Second Edition, Research Studies Press Ltd., 2000

20. A. Sternberger, Experimental video of non-deformable cavity (glass beads - diameter $=2 \mathrm{~mm}$ - high-speed camera - http://umotion.univ-lemans.fr/video/3358-miseen-vibration-dun-volume-de-granulaires-indeformablesdans-une-cavite)

21. G. Michon, Soft hollow particle damping identification in honeycomb structures, Journal of Sound and Vibration, 332, 536 - 544 (2013)

22. E. Grippon, T. Legaud, P.L. Chiambaretto, v. Fascio, Y. Gourinat, V. Lapoujade, Guideline of a metamaterial for launcher tanks dynamic experiments. In: Proceedings of 14th european conference on spacecraft structures, materials \& environmental testing (ECSSMET14) Toulouse, France (2016)

23. P-L. Chiambaretto, Phd Thesis, Modèle vibratoire de réservoir cryotechnique de lanceur: définition d'un métamatériau équivalent, Institut Clément Ader, UMR CNRS $5312(2017)$

24. S.K. Nguyen, P.L. Chiambaretto, M. Charlotte, P. Villedieu, J. Morlier, Y. Gourinat, Towards an analytical formulation for fluid structure tank vibration analysis: Modal equivalency using granular materials, Engineering Structures, 177:345-356 (2018)

25. F. G. Tyzzer, The Properties of Felt in the Reduction of Noise and Vibration, The Journal of the Acoustical Society of America, 19, 872 - 878 (1947)

26. J. I. Dunlop, Felt pad vibration properties and design criteria, The Journal of the Acoustical Society of America, 91, 2696-2702 (1992)

27. A. Sternberger, Experimental video of non-deformable cavity with felt surface (glass beads - diameter $=2 \mathrm{~mm}$ - https://umotion.univ-lemans.fr/video/3455-mise-envibration-dun-volume-de-granulaires-dans-une-cavite-avecfeutrine)

28. V. Denis, A. Pelat, F. Gautier, B. Elie, Modal Overlap Factor of a beam with an acoustic black hole termination, Journal of Sound and Vibration, 333, 24752488, 2014

29. S. Foucaud, G. Michon, Y. Gourinat, A. Pelat, F. Gautier, Artificial cochlea and acoustic black hole travelling waves observation: Model and experimental results, Journal of Sound and Vibration, 333, 3428-3439, 2014

30. E.D. Nobrega, F. Gautier, A. Pelat, J.M.C. Dos Santos, Vibration band gaps for elastic metamaterial rods using wave finite element method, Mechanical Systems and Signal Processing, 79, 192-202, 2016,

31. BIPM, IEC, IFCC, ILAC, ISO, IUPAC, IUPAP and OIML, Guide to the Expression of Uncertainty in Measurement, JCGM 100:2008 (GUM 1995 with minor corrections) (2008) 